\title{
Determination of Anaerobic Threshold by a new approach through the incremental exercise using proportion in heart rate and pulmonary ventilation changes in rowers
}

\author{
Lachezar G. Stefanov ${ }^{\mathrm{ABCD}}$, Svilen E. Nejkov ${ }^{\mathrm{ABCD}}$ \\ National Sports Academy, Bulgaria \\ Authors' Contribution: A - Study design; B - Data collection; C - Statistical analysis; D - Manuscript Preparation; \\ E - Funds Collection
}

\begin{abstract}
Purpose:

The aim of this research is to create a non-invasive and easy to apply in practice approach to determine the anaerobic threshold based only on measurement of the pulmonary ventilation and the hearth rate. It uses proportions, with which these variables were changed during a maximal incremental test.

Material: $\quad$ Twenty athletes from the national rowing team of Bulgaria with average age of 17.5 years were tested. Participants performed a one-time graded incremental exercise test to exhaustion on a rowing ergometer. The proposed new approach for determining the anaerobic threshold is related to detecting the power. Thus, one curve (obtained from differences in percentages of hearth rate and pulmonary ventilation) crosses the other one (obtained from pulmonary ventilation in percentages). The crossing point corresponds to the anaerobic threshold. This approach was compared with two methods determining the lactate threshold, by blood lactate measurement.

Results: $\quad$ The Shapiro-Wilk test results indicated, that the samples of the heart rate of the compared methods have a normal or close to the normal distribution. The Fisher's F-test demonstrated, that the standard deviations of the samples do not differ significantly two by two at $a=0.05$. The Bland\&Altman test presented, that the $95 \%$ of all measurement data points lie within the confidence interval limit for each of the comparisons made between the new approach and two methods.

Conclusions: $\quad$ Our proposed approach is non-invasive and can be easily applied in the field conditions, without using gas-analysing devices. In addition, it is reliable, reproducible and comparable to the accepted for "Gold Standard" methods for determination of anaerobic threshold with $95 \%$ statistical significance.

Keywords: anaerobic threshold, non-invasive, pulmonary ventilation, hearth rate, endurance
\end{abstract}

\section{Introduction}

The management of the training process is important for the effective development of physical performance in endurance sports. For this purpose, objective and informative indicators of the functional state of athletes, are needed. The most commonly used are the following: heart rate (HR); maximal oxygen consumption $\left(\mathrm{VO}_{2 \max }\right)$; the maximal working capacity $\left(\mathrm{W}_{\max }\right)$, and anaerobic threshold (AT). Between 1957 and 1963 Holman [1] introduced the concept of onset of anaerobic metabolism to measure the cardiopulmonary and peripheral aerobic performance capacity. Because the changes of the arterial blood lactate and pulmonary ventilation, they defined this point as the "point of optimal ventilatory efficiency", identical with the " $\mathrm{O}_{2}$ endurance performance limit". A few years later, Wasserman [2] called it the "anaerobic threshold". The term "anaerobic threshold" is defined as an intensity of exercise, involving a large muscle mass, above which measurement of oxygen uptake cannot account for all of the required energy. Stated in other terms, this is the exercise intensity above which there is a net contribution of energy associated with lactate accumulation [3]. In endurance sports, it has been suggested that AT might be a better indicator of aerobic endurance than $\mathrm{VO}_{2 \max }$, as AT may change without changes in $\mathrm{VO}_{2 \max }$. $\mathrm{VO}_{2 \max }$ is a less sensitive indicator to changes in training status than either (o) Lachezar G. Stefanov, Svilen E. Nejkov, 2021

doi:10.15561/26649837.2021.0203 lactate threshold (LT) or ventilatory anaerobic threshold [4]. Gradually the anaerobic threshold AT (expressed in $\% \mathrm{VO}_{2 \max }$ ) has become a standard, because it is closely linked to the $\% \mathrm{VO}_{2 \max }$ that can be maintained in longdistance events. However, it does not represent unique mean to assess aerobic endurance [5]. Many models are described for determining the anaerobic threshold. Ten of them use measurement of the ventilatory and gas exchange response or measurement of blood lactate. Two of them use heart rate (HR) and pulmonary ventilation (Ve) [6]. Related to the power of the work performed, they show characteristic changes linked to the determination of AT.

There are two pathways of energy supply of the muscle contraction: aerobic, when the oxygen is enough and anaerobic, when oxygen is in deficit for metabolic processes. In other words, there is a transition of aerobic to aerobic-anaerobic energy supply. The higher the exercise intensity, the greater the part of anaerobically produced energy. Depending on the physiological features of each individual, this aerobic-anaerobic transition may be steeper or smoother. Because of this, studies over the years have shown, that the research and determination of AT is a difficult process. One of the most commonly used methods for determining the AT is to detect the LT [7]. This is a very accurate method for determining AT, but unfortunately it is invasive. In addition, for a more 
accurate reading, a larger number of blood samples are needed per one test research. Determination of LT can be done visually by determining the Onset of Blood Lactate Accumulation (OBLA) (4 mmol/L) [8] and by $\mathrm{D}_{\max }$ method [9]. Lactate threshold tests can be a valid and reliable alternative to VT and MLSS to identify the workloads at the transition from aerobic to anaerobic metabolism in well-trained runners [10].

A group of methods determine AT by finding the ventilatory threshold (VT). They are attractive because they avoid the unpleasant and sometimes dangerous taking blood samples, which is done repeatedly in one test. VT is defined as the exercise intensity at which the increase in ventilation becomes disproportional to the increase in power output or speed of locomotion during an incremental exercise test [6]. With increasing of the workload, the Ve gradually increases, and after a certain exercise intensity it becomes steeper. The curve of minute Ve shows a curvilinear slope pattern with two break points. The first coincides with the 'aerobic threshold', the second with the 'anaerobic threshold'. The first curvilinear rise in Ve is called the '(first) ventilatory threshold' (VT1). It reflects an increasing ventilatory drive because of excess $\mathrm{CO}_{2}$, stemming from the buffering of lactic acid by bicarbonate. With increasing of workload beyond 'VT' a second curvilinear rise in Ve may be observed. This second increase in ventilatory drive is also caused by increasing acidosis and by additional $\mathrm{CO}_{2}$ stemming from lactic acid buffering [6]. Several ventilatory parameters have been utilised in assessing ventilatory threshold, among which are oxygen consumption, Ve, respiratory exchange ratio (RER), exhaled $\mathrm{CO}_{2}$, and the ratio of ventilation to oxygen consumption $\left(\mathrm{Ve} / \mathrm{VO}_{2}\right)[11,4]$. Detection of the VT is highly subjective, given the fact that different experts can choose different thresholds from the same data, and that it is dependent on the duration of the stages in an incremental test. The non-invasive determination of the AT from ventilatory data during incremental exercise is not reliable [12].

Beaver et al. [13] utilised a computerised regression analysis of the $\mathrm{VCO}_{2}$ versus $\mathrm{VO}_{2}$ slope collected during progressive intensity exercise (V-slope method) to determine AT. Santos and Giannella-Neto [14] compared computerized and visual methods to detect the VT. They found, that the computerized methods for detecting VT are simple to implement and guarantee the reproducibility and repeatability of these measures. The computerized methods showed high reliability when compared to visual inspection. Although the determination of AT by computerized methods for detecting VT is non-invasive, reproducible, and reliable, it requires expensive gas analysis equipment. In most cases, this equipment operated in laboratory conditions with specialized personnel, making it more difficult to apply in daily coaching practice. Nishijima et al. [15] have developed a new objective method of visually estimating VT (by $\mathrm{V}$-slope), assuming that the pre-VT segment is parallel to the respiratory exchange ratio. Thus, determined the VT best agreed with the LT. They have developed a new objective mathematical method to estimate VT that does not require a computer software program.

Another non-invasive approach to determining AT, is measurement of the HR by incremental exercise. A deflection from linearity of the heart rate versus workload curve may be detected. This is a physiological phenomenon observed in approximately $85 \%$ of the healthy adults at the second threshold [6]. It is known as the heart rate deflection point (HRDP). In 1982, Conconi and colleagues suggested that this phenomenon could be used as a non-invasive method to assess the anaerobic threshold. Other authors later failed to find such coincidence between the workload, HRDP, and AT [7, 12]. For example, during an incremental exercise test, in 21 highly trained cyclists, HR response showed a deflection point in $66.7 \%$ of subjects, but was linear in $33.3 \%$ [16]. Even though Conconi Test seems to offer a certain simplicity in its use, in terms of material equipment, it is important to keep in mind the limitations which the use of Conconi Test also involves. The lack of adherence to the standardization requirements, technical issues, and methodology error could have contributed to the undesirable results, to detect HRDP levels in some attempts. Test stability of CT is very low and there is evidence that DP is not $100 \%$ repeated physiology phenomena [17].

Two of the main factors determining oxygen delivery to the muscles are pulmonary ventilation (Ve) and cardiac output $(\mathrm{CO})$. Together they should provide enough amount of oxygenated blood to the muscles. During incremental exercise, they participate in different proportions at different levels of exercise intensity. At lower exercise intensity, the $\mathrm{CO}$ rises more due to an enlargement in stroke volume (SV). As the exercise intensity increases, the $\mathrm{CO}$ continues to rise, but mostly because of an increase in HR and less because of an enlargement in its SV. After a certain intensity, HR increases less. At the same time, at lower levels of exercise intensity Ve increases less, while at a certain level of exercise intensity it starts to increase exponentially. The proportions in the change can be expressed as a percentage of the maximum reached values of both variables (HR and Ve). The percentage expression of one variable makes it possible to compare the dynamics of physiological variables with different dimensions. The difference in proportions will be greatest at a certain exercise intensity during an incremental test, which is probably related to reaching the AT.

The hypothesis we are testing is whether it is possible to create an approach for determining AT based on the proportions by which $\mathrm{HR}$ and Ve change during incremental exercise test.

The aim of the work is to create a non-invasive and easily applicable in practice approach to determine the anaerobic threshold based on the measurement of pulmonary ventilation and heart rate.

\section{Material and Methods}

Participants. Twenty athletes from the national rowing team of Bulgaria with average age of 17.5 years were tested. In this experiment, informed consent was 
obtained from all participants.

Procedure.

Participants performed a one-time graded incremental exercise test to exhaustion on a rowing ergometer. This represents a control test for the effectiveness of the training process of the competitors from the national rowing team. Of all the parameters recorded standardly, in this test we used the indicators for power (W), heart rate (HR), pulmonary ventilation (Ve) and blood lactate (La). Tests were conducted on rowing ergometer system Concept 2, spirometry system Clark C5, and lactate analyser Arkray LactatePro $^{\mathrm{TM}}$. The initial workload was $60 \mathrm{~W}$. Each stage lasted 2 minutes. Each subsequent stage was $40 \mathrm{~W}$ higher than the previous. Blood lactate samples were taken at the end of each stage of the earlobe and were measured by a lactate analyser. Thus, we obtained measurements for each participant for HR, Ve, W and La at the end of each stage.

Approach description.

After the end of the test, we used the peak heart rate $\left(\mathrm{HR}_{\text {peak }}\right)$ of each participant to calculate the percentage of HR achieved (\%HR) for each stage. Thus, we converted the absolute values for HR into percentages. We used the same procedure for pulmonary ventilation as the peak value $\left(\mathrm{Ve}_{\text {peak }}\right)$ was accepted as $100 \%$ of $(\% \mathrm{Ve})$. On the Table 1. is an example for one of the participants. The approach we propose is graphical and the determination of AT was done in a specific sequence.

The difference (Diff) between the percentages of $\% \mathrm{HR}$ and $\% \mathrm{Ve}$ is compared with $\% \mathrm{Ve}$. The $\%$ Ve showed larger and more characteristic changes, and a steeper increase after a certain exercise intensity. This is expressed graphically by the intersection between the Diff and \% Ve curves (Fig. 1). This intersection point has a relation with the point of AT determined by the LT. When constructing curves, it is important to properly scale the axes in the graph. In the main graph on one ordinate the values for $\%$ Ve are plotted, as the minimum is fixed to 1 unit below the smallest percentage of $\% \mathrm{Ve}$. This is the measurement from the end of the first stage. We used it for the lower limit of the scale, because Ve at rest is a very variable indicator, and in addition there is no ventilation with a value of
$0 \%$. Ventilation at the end of the first stage is already a relatively constant indicator and we can set it at the beginning of the scale. This constancy is due to the neurohumoral regulatory mechanisms during exercise. We will give examples of scaling the two ordinates. For instance, a ventilation of $21.08 \mathrm{~L} / \mathrm{min}$ allows us to determine 21 for a minimum scale value for $\% \mathrm{Ve}$. Therefore 21 is $0 \%$ for the $\%$ Ve scale. The upper limit of this scale is also fixed by $\mathrm{Ve}_{\text {peak }}$, which is $100 \%$ for $\% \mathrm{Ve}$. On the other ordinate (at left), the minimum value of the Diff is fixed at 0 . The maximum value is fixed up to 1 unit above the maximum value for Diff. For example, if the highest value for Diff is 36.4 , we need to set a fixed maximum scale limit of 37 . Choosing a higher value than this is not correct, because this indicator is the result of the difference of two 100 percent values. On the abscissa we set the absolute power for each stage. The intersection point between the two curves is very close to the AT determined by LT. Because the intersection point resembles the $\mathrm{X}$ sign, we called the approach to determining AT the X-method. In the end we get the exact power (W) in Watt at which the Diff curve intersects with the \%Ve curve and determine the AT. We then graphically determine the HR corresponding to the absolute power at which AT is detected.

To determine the applicability of our proposed X-method, we compared the AT obtained by it with the AT obtained at OBLA ( $4 \mathrm{mmol} / \mathrm{L})$ and the AT obtained by the $\mathrm{D}_{\max }$ method for determining LT. We compared HR corresponding to each of the three methods for determining AT.

One of the reference methods we focused on is Onset of Blood Lactate Accumulation - OBLA. This approach to determining AT assumes that the anaerobic threshold is the same as the absolute blood lactate concentration of 4 $\mathrm{mmol} / \mathrm{L}$, and is described by Mader et al. in [18]. OBLA is usually measured in tests of increasing intensity and subsequent interpolation to determine the intensity of the exercise that is expected to cause $4 \mathrm{mmol} / \mathrm{L}$ blood lactate. The advantage of using $4 \mathrm{mmol} / \mathrm{L}$ lactate as a criterion for OBLA assessment is that it offers very objective assessment of the lactate threshold. Another advantage is

Table 1. Displays the raw data for one of the participants.

\begin{tabular}{|c|c|c|c|c|c|c|}
\hline $\mathbf{w}$ & La & HR & Ve & $\% \mathrm{HR}_{\text {peak }}$ & $\% \mathrm{Ve}$ & Diff \\
\hline 60 & 1.1 & 102 & 37.2 & 53.4 & 21.1 & 32.3 \\
\hline 100 & 1.1 & 115 & 49.4 & 60.2 & 28.0 & 32.2 \\
\hline 140 & 1.2 & 125 & 57.9 & 65.4 & 32.8 & 32.6 \\
\hline 180 & 1.4 & 143 & 67.8 & 74.9 & 38.4 & 36.4 \\
\hline 220 & 1.4 & 158 & 84.5 & 82.7 & 47.9 & 34.8 \\
\hline 260 & 1.8 & 174 & 101.6 & 91.1 & 57.6 & 33.5 \\
\hline 300 & 3.1 & 181 & 118.1 & 94.8 & 67.0 & 27.8 \\
\hline 340 & 4.1 & 188 & 136.3 & 98.4 & 77.3 & 21.2 \\
\hline 380 & 6.6 & 176 & 158.1 & 92.1 & 89.6 & 2.5 \\
\hline 420 & 8.4 & 191 & 176.4 & 100.0 & 100.0 & 0.0 \\
\hline
\end{tabular}

NOTE: In the columns La, HR, Ve, W, and derivative values in the columns \%HR ${ }_{\text {peak }}$ \%Ve, and Diff. 
that $4 \mathrm{mmol} / \mathrm{L}$ is significantly higher than the resting level, which can be quite variable. This means, that $4 \mathrm{mmol} / \mathrm{L}$ will represent a fairly narrow range of intensity during a test with increasing intensity. The problem with the use of absolute concentration of blood lactate is the insensitivity to individual physiological differences [5].

$\mathrm{D}_{\text {max }}$ is the other reference method for determining AT by LT. First proposed by Cheng [19] LT is defined as the largest perpendicular distance from the line constructed between the first and last lactate measurement (lactate straight line) under increased exercise, and a point on the curve of a 3 degree polynomial that represents the changes in the lactate during the test. This point reflects the change in the tendency of the blood lactate during exercise. The accuracy of the method depends on the location of the first and last data point from the lactate curve, because of which it shows some variability. To overcome this influence, Bishop et al. [9] proposes a modification of the method. According to him, the lactate straight line starts from the point preceding the first measured difference of $0.4 \mathrm{mmol} / \mathrm{L}$ between two consecutive measurements and ends at the last measurement. In order to refine the determination of LT by this modified $\mathrm{D}_{\max }$ method, we constructed a line, parallel to the lactate straight line, which passes as tangent to the curve of the third degree polynomial (Fig. 2). The position of contact between them determined the point from which a perpendicular is descended to the lactate straight line. For each of the participants, we plotted a graph, with La scaled on the ordinate and the power of each stage on the abscissa

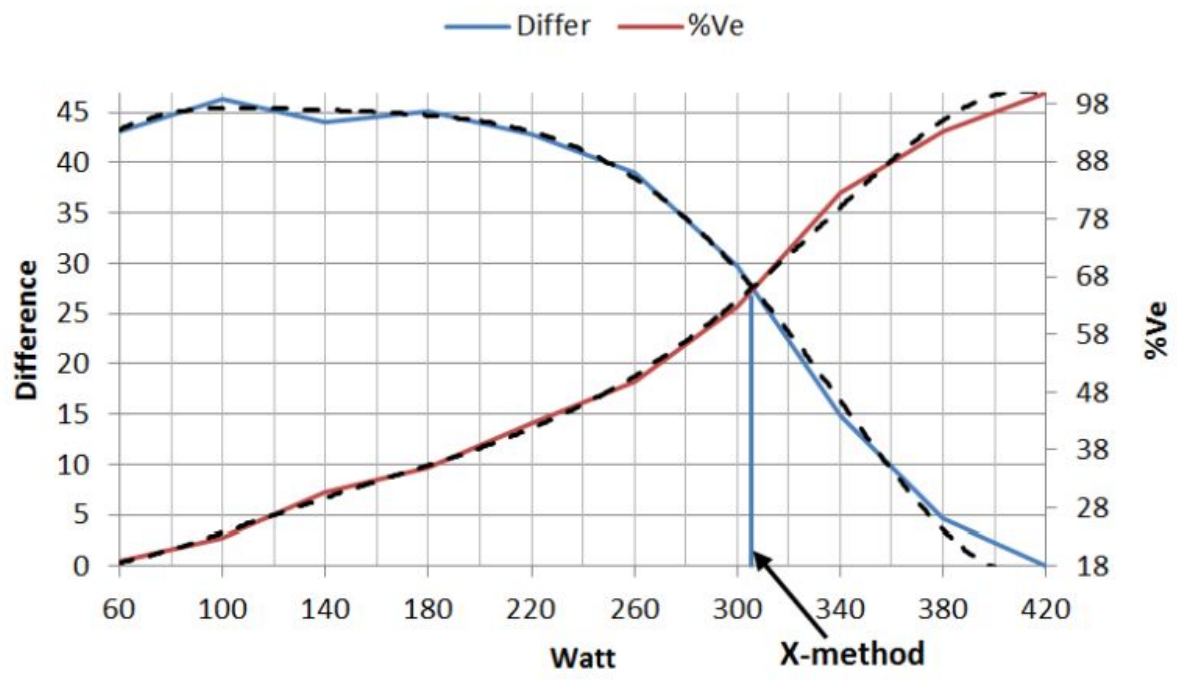

Figure 1. Reflects the relationship between \%Ve and the difference between \%HR and \%Ve. A dotted line shows the 5-degree polynomials of both variables. The intersection point between the curves of the two polynomials determines the AT by the X-method.

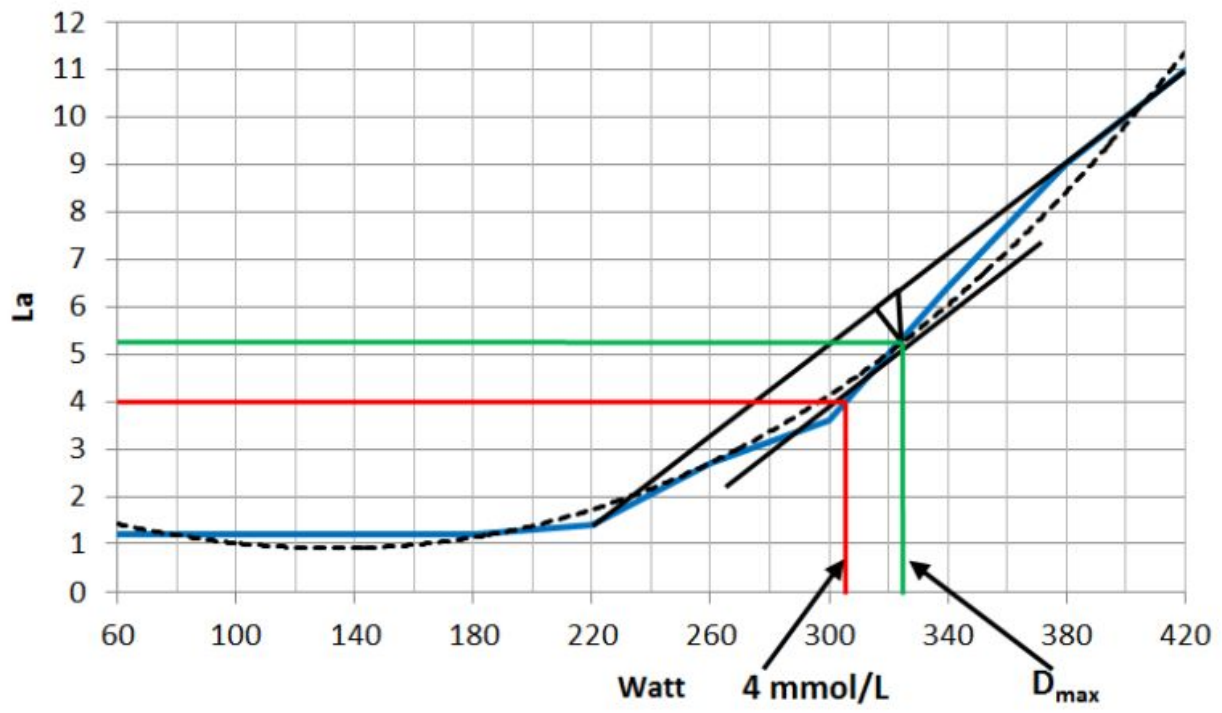

Figure 2. Represents the determination of the power corresponding to the AT determined by the $4 \mathrm{mmol} / \mathrm{L} \mathrm{method}$ and the AT determined by the modified Dmax method. 


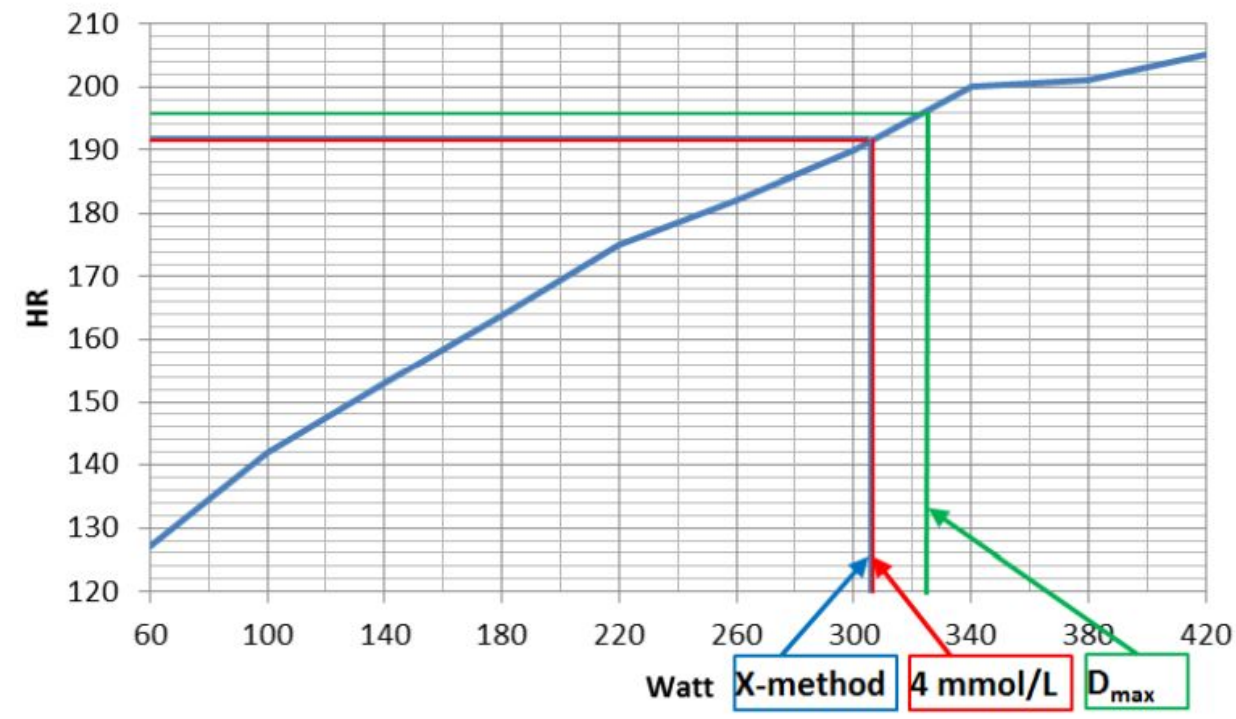

Figure 3. Represents HR corresponding to the power at which AT is detected by each of the three methods.

(Fig. 2). According to this graph, we defined AT as power corresponding to $4 \mathrm{mmol} / \mathrm{L}$ and AT by the modified $\mathrm{D}_{\max }$ method.

To determine the HR versus the power corresponding to the AT determined by each of the three methods, we used a graphical approach (Fig. 3)

Statistical analysis.

To compare the three methods, we arranged the HR corresponding to the AT determined by the respective method for each participant (Table 2). Then we did a test for normality of the distribution of HR for each of the three methods. We used the Shapiro-Wilk test. For comparison of the standard deviations in normally distributed data or close to the normal distribution, we used Two-tailed Fisher's F-test. If the standard deviations of two samples do not differ statistically, it can be assumed that they belong to one general sample. To compare the new approach with the two classic methods, we used the Bland \& Altman test, which offers a simple parametric approach based on dispersion analysis and simple graphical methods [20]. The Bland - Altman (B\&A) diagram describes the consistency between two quantitative measurements. The area of consistency is calculated by the median and standard deviation of the differences between the two measurements. B\&A recommends that $95 \%$ of the points lie within limits of $\pm 2 \mathrm{~s}$ from the median difference ( $\mathrm{s}$ - standard deviation). This way of applying the B\&A method is the most common one [21].

For statistical data processing we used EXCEL 10 of Microsoft Office 10 and the application XLSTAT developed for the purpose.

\section{Results}

Our proposed X-method was compared with two approved methods for determining AT based on the concentration of blood lactate.
Table 2. Represents the HR measured at AT determined by the respective method.

\begin{tabular}{llll}
\hline Subject & $\mathbf{D}_{\max }$ & X-method & $\mathbf{4 m m o l} \mathbf{L}$ \\
\hline $\mathbf{1}$ & 190.00 & 190.00 & 190.00 \\
$\mathbf{2}$ & 180.00 & 177.00 & 177.00 \\
$\mathbf{3}$ & 174.00 & 173.00 & 171.00 \\
\hline $\mathbf{4}$ & 185.00 & 183.00 & 185.00 \\
$\mathbf{5}$ & 201.00 & 196.00 & 196.00 \\
\hline $\mathbf{6}$ & 187.00 & 185.00 & 180.00 \\
$\mathbf{7}$ & 186.00 & 184.00 & 185.00 \\
\hline $\mathbf{8}$ & 179.00 & 176.00 & 177.00 \\
\hline $\mathbf{9}$ & 174.00 & 170.00 & 175.00 \\
\hline $\mathbf{1 0}$ & 177.00 & 177.00 & 179.00 \\
\hline $\mathbf{1 1}$ & 192.00 & 188.00 & 190.00 \\
\hline $\mathbf{1 2}$ & 179.00 & 176.00 & 179.00 \\
\hline $\mathbf{1 3}$ & 178.00 & 173.00 & 176.00 \\
\hline $\mathbf{1 4}$ & 173.00 & 172.00 & 168.00 \\
\hline $\mathbf{1 5}$ & 191.00 & 191.00 & 191.00 \\
\hline $\mathbf{1 6}$ & 181.00 & 179.00 & 181.00 \\
\hline $\mathbf{1 7}$ & 190.00 & 190.00 & 188.00 \\
\hline $\mathbf{1 8}$ & 196.00 & 191.00 & 191.00 \\
\hline $\mathbf{1 9}$ & 174.00 & 171.00 & 168.00 \\
\hline $\mathbf{2 0}$ & 186.00 & 186.00 & 188.00 \\
\hline $\mathbf{S T D E V}$ & 7.995 & 7.956 & 8.097 \\
\hline Aver. & 183.65 & 181.40 & 181.75 \\
\hline & & & \\
\hline
\end{tabular}

NOTE: In the first column are the consecutive numbers of the participants in the experiment. In the second column are the HR for AT determined by the $D_{\text {max }}$ method, in the third are HR for AT determined by the X-method, in the fourth are HR for AT determined at $4 \mathrm{mmol} / \mathrm{L}$. 
On the Table 2 are shown the HRs corresponding to AT determined by each of the respective methods. The average HR for each of the three methods differ slightly from each other. Furthermore, the same is observed for the standard deviations, which are also slightly different compared to one another. This indicates that the methods probably have a similar ability to determine AT. From Table 2 can be seen that the standard deviations of the HR of the three methods for determining AT and their averages are very close. Therefore, for the three samples from the table a test for normality of distribution should be performed. The results of the Shapiro-Wilk test are presented in Table 3 and show that all three samples for HR have a normal or close to the normal distribution. This allows us to choose parametric methods to compare the three methods. If the differences between their standard deviations and their average values are not statistically significant, then they can be applied alternatively.

Table 3. Represents $p$-value for each of the three samples, with statistical significance $a=0.05$

\begin{tabular}{ll}
\hline Variable & Shapiro-Wilk p-value \\
\hline$\left(D_{\max }\right)$ & 0.307 \\
$(X-m e t h o d)$ & 0.199 \\
$(4 \mathrm{mmol} / \mathrm{L})$ & 0.493 \\
\hline
\end{tabular}

Fisher's F-test showed that the standard deviations of the samples two by two do not differ significantly at $\mathrm{a}=$ 0.05 , and therefore, belong to the same group (Table 4).

Table 4. Represents p-value for Fisher's F-test for comparing the standard deviations of the three methods for determining AT.

\begin{tabular}{ll}
\hline Variable & Fisher p-value \\
\hline X-method vs. $D_{\max }$ & 0.983 \\
X-method vs. 4mmol/L & 0.940 \\
$D_{\max }$ vs. 4mmol/L & 0.975 \\
\hline
\end{tabular}

On the Table 5 are presented the results of the analysis of the B\&A test for the three methods for determining AT, each compared with the others. Heart rates for AT determined by the three methods are represented graphically by the B\&A method in Figure 4 . There are compared between each other all three methods for determining the AT.
It is seen, that $95 \%$ of all measurements lie within the confidence interval, for each comparison of a method with the others. As shown on table 5, the $\mathrm{D}_{\max }$ method reveals AT at higher HR having 2.25 average heartbeats per minute more, in comparison to the X-method. $4 \mathrm{mmol} / \mathrm{L}$ method reveals AT at higher HR with 0.35 average heartbeats per minute more, in comparison to the $\mathrm{X}$-method. When we compare the two approved methods, we can also see a difference in the HR in determining AT. This difference shows that the $4 \mathrm{mmol} / \mathrm{L}$ method reveals AT at lower HR having 1.9 average heartbeats per minute less in comparison to the $\mathrm{D}_{\max }$ method. The standard error from the three comparisons is the lowest when comparing the X-method with the $\mathrm{D}_{\max }$.

\section{Discussion}

The comparison between the three methods for determining of AT shows that the differences between them are not statistically significant. The advantage of the proposed from us approach (X-method) is, that it requires only a Hearth Rate Monitor, Portable Device for measuring Ve, and Device for measuring Movement Speed or Power of Work. Twelve models are used to determine AT [6], each of which has its advantages and disadvantages. We will mention some of the basic models with their disadvantages, that are avoided with our approach. Using a fixed lactate value as the threshold certainly increases objectivity but denies individuality since the non-linear increase in blood lactate does not always occur at $4 \mathrm{mmol} / \mathrm{L}$ [5]. In addition, the procedure is invasive. Moreover, even if we assume that all studies are using the same definition and the same criteria to detect the LT, there is strong evidence to suggest that these thresholds do not occur at the same power output [5]. One of the most accurate methods for determining $\mathrm{LT}$ is the modified $\mathrm{D}_{\max }$ method described above, but unfortunately it is invasive. On the other hand, the level of AT determined with the Dmax method significantly differs in comparison to the level of V-slope method [22], which is not discussed in this article, but is one of the basic non-invasive methods for determining of AT. However, the V-slope method requires expensive laboratory equipment and personnel. It can also be performed in the field conditions by using expensive portable gas analysers, which have less measurement accuracy than laboratory ones.

One non-invasive, easy for use and inexpensive method for determining AT is the detection of the heart

Table 5. Bias - average of the differences from one compared method - (minus) the other compared method; Standard error; $\mathrm{Cl}$ Bias - deviation range; Confidence interval of differences.

\begin{tabular}{llll}
\hline Statistical parameter & X-method vs. $\mathbf{D}_{\max }$ & X-method vs. 4mmol/L & D $_{\max }$ vs. 4mmol/L \\
\hline Bias & 2.25 & 0.35 & -1.9 \\
Standard error & 1.773 & 2.519 & 2.634 \\
Cl Bias (95\%) & $1.420 \div 3.080$ & $-0.829 \div 1.529$ & $-3.133 \div-0.667$, \\
$\begin{array}{l}\text { Confidence interval } \\
\text { (Differences): }\end{array}$ & $-1.226 \div 5.726$ & $-4.587 \div 5.287$ & $-7.062 \div 3.262$, \\
\hline
\end{tabular}



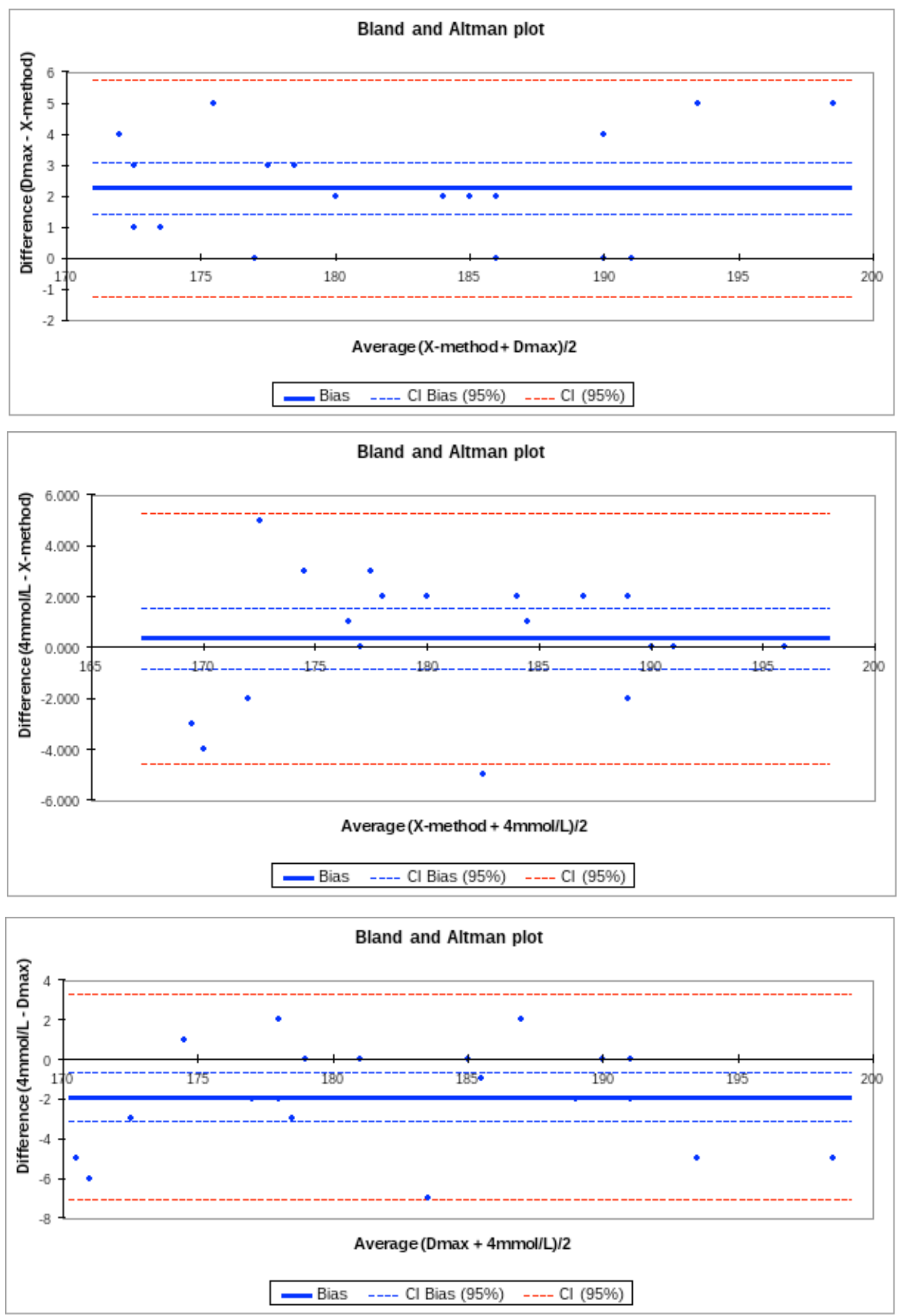

Figure 4. Displays the data points of the compared methods within the confidence interval by the Bland-Altman method.

rate deflection point (HRDP) described by Conconi et al. [23]. Despite the fact that the Conconi test is used by some athletes and coaches, there is serious criticism of the method in the scientific literature. The main difficulty comes from the concept of the deflex point, as some studies have failed to find it systematically in all investigated subjects. Therefore, a number of authors question the physiological existence of HRDP and suggest that it is a protocol-dependent artefact [24, 5]. Jones and Doust [25] concluded that the failure to determine 
repeatable HR deviation in the assessment in 9 of the 15 examined subjects makes the Conconi test unsuitable for reliable assessment of AT. The controversy over the use of HRDP to assess the anaerobic threshold may be related to differences in methodologies. HRDP appears to be associated with LT, but many researches are still needed to link to the maximum lactate steady state (MLSS) [5]. We did not mention MLSS, although it is a very accurate method for determining AT. Unfortunately, it is invasive with a very long and precise procedure. In other hand traditional $\mathrm{D}_{\max }$ and OBLA of 4.0 mmol. $\mathrm{L}^{-1}$ methods did not provide valid estimates of the MLSS [26].

There are other attempts to use HR to determine AT. By laboratory trials, the measured HR and power output at the AT, determined either by respiratory gas indices or the HRDP method, give comparable results with AT determined by blood lactate concentrations and could, therefore, be used for guiding the appropriate intensity of rowing training [27].

\section{Conclusions}

Our proposed approach is non-invasive and can be easily applied in field conditions, without using gasanalysing devices. In addition, it is reliable, reproducible and comparable to the accepted for "Gold Standard" methods for determination of anaerobic threshold with $95 \%$ statistical significance. We have not found similar methods using only heart rate, pulmonary ventilation and speed movement or power of work in the literature available to us, therefore we consider this approach to be new to the sports practice. To be able this approach to be used more widely in sports practice, research in other endurance sports is needed. It could also be used in clinical trials to monitor the physical condition of various contingents of subjects.

\section{Conflict of interest}

The authors declare no conflict of interest.

\section{References}

1. Holman.W. Historical Remarks on the Development of the Aerobic-Anaerobic Threshold up to 1966. Int. J. Sports Med. 1985; 6:109-116. https://doi.org/10.1055/s-2008-1025823

2. Wasserman K, Whipp B, Koyal S, Beaver B. Anaerobic threshold and respiratory gas exchange during exercise. J. Appl. Physiol. 1973; 35(Z): 236-243. https://doi.org/10.1152/jappl.1973.35.2.236

3. Svedahl K, MacIntosh BR. Anaerobic threshold: The concept and methods of measurement. Can. J. Appl. Physiol. 2003; 28(2): 299-323. https://doi.org/10.1139/h03-023

4. Ghosh AK. Anaerobic Threshold: Its Concepts and Role in Endurance Sport. Malaysian Journal of Medical Sciences. 2004; 11 (1):24-36

5. Bosquet L, Léger L Legros P. Methods to Determine Aerobic Endurance. Sports Med. 2002; 32 (11): 675-700. https://doi.org/10.2165/00007256-200232110-00002

6. Bindera RK, Wonischb M, Corrac U, Cohen-Solald A, Vanheese L, Sanera Hetal. Methodological approach to the first and second lactate threshold in incremental cardiopulmonary exercise testing. European Journal of Cardiovascular Prevention and Rehabilitation. 2008; 15:726-734. https://doi.org/10.1097/HJR.0b013e328304fed4

7. Billat VL. Use of Blood Lactate Measurements for Prediction of Exercise Performance and for Control of Training Recommendations for LongDistance Running. Sports.Med. 1996;22 (3):157-175. https://doi.org/10.2165/00007256-199622030-00003

8. Heck H, Mader A, Hess G, Mücke S, Müller R, Hollmann W. Justification of the 4-mmol/l lactate threshold. Int $J$ Sports Med. 1985; 6(3):117-130. https://doi.org/10.1055/s-2008-1025824

9. BishopD,JenkinsDG,MackinnonLT.Therelationshipbetween plasmalactate parameters, Wpeak and 1-h cycling performance in women. Med Sci Sports Exerc. 1998;30(8):1270-1275. https://doi.org/10.1097/00005768-199808000-00014

10.Cerezuela-Espejo V, Courel-Ibáñez J, Morán-Navarro R, Martínez-Cava A, Pallarés JG. The Relationship Between Lactate and Ventilatory Thresholds in Runners: Validity and Reliability of Exercise Test Performance Parameters. Front. Physiol. 2018;9:1320. https://doi.org/10.3389/fphys.2018.01320

11.Gaskill SE, Ruby BC, Walker AJ, Sancez OA, Serfass RC, Leon AS. Validity and reliability of combining three methods to determine ventilatory threshold. Med. Sci. Sports Exerc. 2001; 33,11:1841-1848. https://doi.org/10.1097/00005768-200111000-00007

12.Bodner ME, Rhodes C. A review of the concept of the heart rate deflection point. Sports Med. 2000;30,1:31-46. https://doi.org/10.2165/00007256-200030010-00004

13.Beaver W, Wasserman K, Whipp BJ. A new method for detecting anaerobic threshold by gas exchange. $J$ Appl Physiol 1986; 60: 2020-7.

14.Santos EL, Giannella-Neto A. Comparison of computerized methods for detecting the ventilatory thresholds. Eur J Appl Physiol. 2004; 93: 315-324. https://doi.org/10.1007/s00421-004-1166-6

15.Nishijima H, Kominami K, Kondo K, Akino M, Sakurai M. New method for the mathematical derivation of the ventilatory anaerobic threshold: a retrospective study. $B M C$ Sports Science, Medicine and Rehabilitation. 2019; 11:10. https://doi.org/10.1186/s13102-019-0122-z

16.Lucía A, Carvajal A, Boraita A, Serratosa L, Hoyos J, Chicharro JL. Heart dimensions may influence the occur rence of the hear $t$ rate deflection point in highly trained cyclists. $\mathrm{Br}$ J Sports Med. 1999;33:387-392. https://doi.org/10.1136/bjsm.33.6.387

17.Hnizdil J, Skopek M, Nosek M, Louka O, Musalek M, Heller J. The Conconi Test - Searching for the Deflection Point. Physical Activity Review. 2019; 7: 160-167. https://doi.org/10.16926/par.2019.07.19

18. Mader A, Liesen H, Heck H, Philippi H, Rost R, Schürch $\mathrm{P}$ et al. Zur Beurteilung der sportartspezifischen usdauerleistungsfähigkeit im Labor. [To assess the sportspecific endurance capacity in the laboratory]. Sportarzt und Sportmedizin, 1976; 27(5):109-112. (In German)

19.Cheng BK, Snyder H, Keizer AC, Jeukendrup HA, Hesselink M. A new approach for the determination of ventilatory and lactate thresholds. Int J Sports Med. 1992;13: 518-522. https://doi.org/10.1055/s-2007-1021309

20.Altman DG, Bland JM. Measurement in Medicine: the Analysis of Method. The Statistician. 1983;32:307-317. https://doi.org/10.2307/2987937 
21.Giavarina D. Understanding Bland Altman analysis. Biochemia Medica. 2015;25(2):141-51. https://doi.org/10.11613/BM.2015.015

22.Strzala M, Tyka A, Zychowska M. Reliability of Invasive ad Non-Invasive Anaerobic Threshold Estimation in Young Swimmers. Journal of Human Kinetics volume. 2004;12:135-146.

23.Conconi F, Ferrari M, Ziglio PG, Droghetti P, Codeca L: Determination of the anaerobic threshold by a noninvasive field test in runners. J. Appl. Physiol. Respir. Environ. Exerc. Physiol. 1982;52: 869-873. https://doi.org/10.1152/jappl.1982.52.4.869

24.Bourgois J, Coorevits P, Danneels L, Witvrouw E, Cambier D, Vrijens J. Validity of the heart rate deflection point as a predictor of lactate threshold concepts during cycling. $J$ Strength Cond res. 2004;18(3):498-503. https://doi.org/10.1519/00124278-200408000-00018

25.Jones A, Doust J. Lack of reliability in Conconi's heart rate deflection point. Int. J. Sports Med. 1995; 16: 541-544. https://doi.org/10.1055/s-2007-973051

26.Jamnick NA, Botella J, Pyne DB, Bishop DJ. Manipulating graded exercise test variables affects the validity of the lactate threshold and VO2peak. PLOS ONE. 2018; 13(7): e0199794 https://doi.org/10.1371/journal.pone.0199794

27.Erdogan A, Cetin C, Karatosun H, Baydar M. Non-invasive Indices for the Estimation of the Anaerobic Threshold of Oarsmen. The Journal of International Medical Research. 2010; 38: 901 -915. https://doi.org/10.1177/147323001003800316

\section{Information about the authors:}

Lachezar G. Stefanov; (Corresponding Author); http://orcid.org/0000-0001-5380-3446; luchos@nsa.bg; Department Physiology and Biochemistry, National Sports Academy ; Sudentski Grad, Acad. Stefan Mladenov Str. N-21, PC:1710 Bulgaria, Sofia.

Svilen E. Nejkov; http://orcid.org/0000-0002-3336-0454; svilen.neykov@abv.bg; Department - Theory of Sport, National Sports Academy; Sudentski Grad, Acad. Stefan Mladenov Str. N-21, PC:1710, Bulgaria, Sofia.

Cite this article as:

Stefanov LG, Nejkov SE. Determination of Anaerobic Threshold by a new approach through the incremental exercise using proportion in heart rate and pulmonary ventilation changes in rowers. Pedagogy of Physical Culture and Sports, 2021;25(2):89-97.

https://doi.org/10.15561/26649837.2021.0203

This is an Open Access article distributed under the terms of the Creative Commons Attribution License, which permits unrestricted use, distribution, and reproduction in any medium, provided the original work is properly cited (http://creativecommons.org/licenses/by/4.0/deed.en).

Received: 10.07.2020

Accepted: 25.08.2020; Published: 30.04.2021 